\title{
On the relation between length functions and exact Sylvester rank functions
}

https://doi.org/10.1515/taa-2019-0006

Received February 28, 2019; accepted July 15, 2019

Abstract: Inspired by the work of Crawley-Boevey on additive functions in locally finitely presented Grothendieck categories, we describe a natural way to extend a given exact Sylvester rank function on the category of finitely presented left modules over a given ring $R$, to the category of all left $R$-modules.

Keywords: Sylvester rank function, length function, additivity, extension of invariants, matrix rank function

2010 MSC: Primary 16D10, Secondary 16E50, 16E20

\section{Introduction}

The notion of Sylvester matrix rank function has been studied and applied in different areas of Algebra, especially in the context of $C^{\star}$-algebras and von Neumann regular rings. In fact, these functions were already considered by von Neumann, and they were investigated by I. Halperin, K. R. Goodearl and D. Handelman, among others, see [3] for an exposition of these results and classical references for the theory. For general rings, they were introduced by Malcolmson in order to characterize ring homomorphisms to division rings and simple artinian rings, we refer to [6] for results going in this direction. Let us remark that, in this theory, there is a special class of rank functions called exact Sylvester rank functions that correspond to flat embeddings.

There has been a recent trend of studies on Sylvester rank functions, in connection, amongst other subjects, with the strong Atiyah Conjecture and the Lück approximation Conjecture, see [4] for a survey on these results.

A Sylvester matrix rank function $\rho$ on a (associative and unitary) ring $R$ is a function that assigns a non-negative real number to each matrix over $R$ and satisfies the following conditions:

(Mat.rk.1) $\rho(A)=0$ if $A$ is a zero matrix and $\rho(1)=1$;

(Mat.rk.2) $\rho(A B) \leq \min \{\rho(A), \rho(B)\}$ for any matrices $A$ and $B$ which can be multiplied;

(Mat.rk.3) $\rho\left(\begin{array}{cc}A & 0 \\ 0 & B\end{array}\right)=\rho(A)+\rho(B)$ for any matrices $A$ and $B$;

(Mat.rk.4) $\rho\left(\begin{array}{ll}A & B \\ 0 & C\end{array}\right) \geq \rho(A)+\rho(C)$ for any matrices $A, B$ and $C$, of appropriate sizes.

Another, closely related, approach to rank functions is that of Sylvester module rank functions. Indeed, such a function is a non-negative real invariant defined on the category of finitely presented left $R$-modules $\delta: \operatorname{fp}(R) \rightarrow \mathbb{R}_{\geq 0}$, that satisfies the following axioms:

(Mod.rk.1) $\delta(0)=0$ and $\delta(R)=1$;

(Mod.rk.2) $\delta(P \oplus Q)=\delta(P) \oplus \delta(Q)$, for all $P$ and $Q \in \mathrm{fp}(R)$;

(Mod.rk.3) if $P \rightarrow Q \rightarrow S \rightarrow 0$ is exact in $\mathrm{fp}(R)$, then $\delta(P)+\delta(S) \geq \delta(Q) \geq \delta(S)$.

^Corresponding Author: Simone Virili: Universidad de Murcia Murcia, Murcia Spain, E-mail: virili.simone@gmail.com 
It is an easy but useful exercise to verify that, given a Sylvester matrix rank function $\rho$ on $R$ one obtains a Sylvester module rank function $\delta_{\rho}$ on the same ring just letting $\delta_{\rho}(P):=\rho\left(A_{P}\right)$, where $A_{P}$ is the matrix associated with a given presentation of $P$. On the other hand, given a Sylvester module rank function $\delta$, one obtains a Sylvester matrix rank function $\rho_{\delta}$ just letting $\rho_{\delta}(A):=\delta\left(R^{n} / R^{m} A\right)$, where we have interpreted the matrix $A$ as a morphism $R^{m} \rightarrow R^{n}$. One can show that these procedures induce a bijection between Sylvester matrix and module rank functions, so the two theories are essentially equivalent.

A Sylvester module rank function $\chi: \mathrm{fp}(R) \rightarrow \mathbb{R}_{\geq 0}$ is said to be exact if it satisfies the following condition: (EX) given a surjection $X \rightarrow Y$ in $\mathrm{fp}(R)$,

$$
\chi(X)-\chi(Y)=\inf \{\chi(K): K \in \mathrm{fp}(R), K \rightarrow \operatorname{Ker}(\phi)\} .
$$

The present paper originates from a question asked me by Andrei Jaikin in 2017 during a visit to the ICMAT in Madrid. In particular, it is known for some special classes of rings (e.g., von Neumann regular rings) that any exact Sylvester module rank function $\delta: \mathrm{fp} R \rightarrow \mathbb{R}_{\geq 0}$ can be extended to a very well-behaved (i.e., additive, see below) invariant $\bar{\delta}: R$-Mod $\rightarrow \mathbb{R}_{\geq 0} \cup\{\infty\}$. Thus, it is natural to ask for a characterization of the class of rings where any exact Sylvester module rank function is the restriction to finitely presented modules of an additive function defined on all modules.

Some time after this question was asked to me I recalled that a similar problem, with different terminology, had been studied in the setting of locally finitely presented Grothendieck categories by Crawley-Boevey $[1,2]$ and, in fact, minor modifications of Crawley-Boevey's argument (essentially due to the fact that CrawleyBoevey's functions take values in $\mathbb{N}$ while our invariants are real-valued) allow us to prove the following

Main Theorem. Given an exact Sylvester module rank function $\chi: \mathrm{fp}(R) \rightarrow \mathbb{R}_{\geq 0}$, there exists a function $\widetilde{\chi}: R$-Mod $\rightarrow \mathbb{R}_{\geq 0} \cup\{\infty\}$ which extends $\chi\left(\right.$ i.e., $\left.\widetilde{\chi} \uparrow_{\mathrm{fp}(R)}=\chi\right)$ and which is additive in the following sense: (ADD) given $N \leq M$ in $R$-Mod, $\widetilde{\chi}(M)=\widetilde{\chi}(N)+\widetilde{\chi}(M / N)$.

In fact, it will be clear from the construction that the function $\widetilde{\chi}: R$-Mod $\rightarrow \mathbb{R}_{\geq 0} \cup\{\infty\}$ produced by the above theorem is a length function in the sense of $[5,7,8]$. As a final remark, let us notice that all the results in this short paper can be trivially extended to locally finitely presented Grothendieck categories but we preferred to write this note in the setting of modules over a ring, as it is the setting where the most applications of this formalism are expected.

\section{Proof of the Main Theorem}

This entire section is devoted to the proof of our Main Theorem. This proof proceeds by steps: in Subsection 1.1 we extend our exact function from the class of finitely presented left $R$-modules $\mathrm{fp}(R)$ to the class of finitely generated left $R$-modules $\mathrm{fg}(R)$, while in Subsection 1.2 we extend it from $\mathrm{fg}(R)$ to $R$-Mod. Finally, in the last two subsections, we prove that the invariant of $R$-Mod obtained via this extension procedure is additive, so it gives us a length function.

\subsection{Extension to $\mathrm{fg}(R)$}

Let us define a function

$$
\bar{\chi}: \operatorname{fg}(R) \rightarrow \mathbb{R}_{\geq 0}
$$

on a finitely generated module $F \in \operatorname{fg}(R)$ by the following formula:

$$
\bar{\chi}(F):=\inf \{\chi(X): X \rightarrow F, X \in \operatorname{fp}(R)\} .
$$


Notice first that, given $X \in \mathrm{fp}(R)$, then $\mathrm{id}_{X}: X \rightarrow X$ is a surjection from a finitely presented module, so $\bar{\chi}(P)=\chi(P)$. This shows that $\bar{\chi} \uparrow_{\mathrm{fp}(R)}=\chi$.

We collect in the following lemma some easy observations about $\bar{\chi}$. In particular, we show that $\bar{\chi}$ is monotone under taking quotients and finitely generated submodules:

Lemma 1.1. In the above setting, the following properties hold true:

(1) if $F \rightarrow F^{\prime}$ in $\mathrm{fg}(R)$, then $\bar{\chi}\left(F^{\prime}\right) \leq \bar{\chi}(F)$;

(2) given a short exact sequence $0 \rightarrow F \rightarrow X \rightarrow Y \rightarrow 0$ with $X, Y \in \mathrm{fp}(R)$, then $F \in \operatorname{fg}(R)$ and $\bar{\chi}(X)=\bar{\chi}(F)+\bar{\chi}(Y)$;

(3) given $F^{\prime} \leq F$ in $\mathrm{fg}(R)$, then $\bar{\chi}\left(F^{\prime}\right) \leq \bar{\chi}(F)$

Proof. Part (1) follows directly from the definition since, given a surjection $X \rightarrow F$, composition with $F \rightarrow F^{\prime}$ gives a surjection $X \rightarrow F^{\prime}$. Now consider a short exact sequence as in part (2). The fact that $F$ is finitely generated is an easy consequence of Schanuel's Lemma. But then, by exactness and the observation that $\bar{\chi} \uparrow_{\mathrm{fp}(R)}=\chi$, we obtain:

$$
\bar{\chi}(X)-\bar{\chi}(Y)=\chi(X)-\chi(Y)=\inf \{\chi(K): K \in \mathrm{fp}(R), K \rightarrow F\}=\bar{\chi}(F) .
$$

Let us now verify part (3). Indeed, given $\varepsilon>0$, consider a surjection $X \rightarrow F$, with $X \in \mathrm{fp}(R)$, such that $\chi(X) \leq \bar{\chi}(F)+\varepsilon$, and complete $X \rightarrow F \leftarrow F^{\prime}$ to the following pullback diagram:

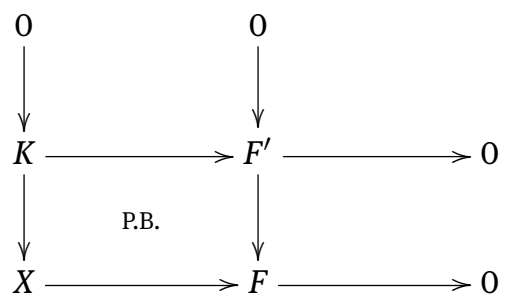

where rows and columns are exact. Then, since $F^{\prime}$ is finitely generated, there exists a finitely generated submodule $K^{\prime} \leq K$ such that the restriction $K^{\prime} \rightarrow F^{\prime}$ is still surjective. Furthermore, after identifying $K$ and $K^{\prime}$ with submodules of $X$, we can notice that $X / K^{\prime}$ is finitely presented. Now we conclude as follows:

$$
\bar{\chi}\left(F^{\prime}\right) \leq \bar{\chi}\left(K^{\prime}\right)=\bar{\chi}(X)-\bar{\chi}\left(X / K^{\prime}\right) \leq \chi(F)+\varepsilon,
$$

where the central equality holds by part (2). As the choice of $\varepsilon$ was arbitrary, we deduce that $\bar{\chi}\left(F^{\prime}\right) \leq \bar{\chi}(F)$.

\subsection{Extension to $R$-Mod}

Let us define a function

$$
\widetilde{\chi}: R-\operatorname{Mod} \rightarrow \mathbb{R}_{\geq 0} \cup\{\infty\}
$$

on a module $M \in R$-Mod by the following formula:

$$
\widetilde{\chi}(M):=\sup \{\bar{\chi}(F): F \leq M, F \in \mathrm{fg}(R)\} .
$$

Notice first that, given $F \in \operatorname{fg}(R)$, then $F \leq F$ is a finitely generated submodule of itself, and so, by the monotonicity of $\bar{\chi}$ under taking finitely generated submodules, $\widetilde{\chi}(F)=\bar{\chi}(F)$. This shows that $\widetilde{\chi} \uparrow_{\mathrm{fg}(R)}=\bar{\chi}$ and so, in particular, also that

$$
\tilde{\chi} \uparrow_{\mathrm{fp}(R)}=\bar{\chi} \uparrow_{\mathrm{fp}(R)}=\chi
$$

\section{$1.3 \widetilde{\chi}$ is super-additive}

Consider a short exact sequence in $R$-Mod as follows:

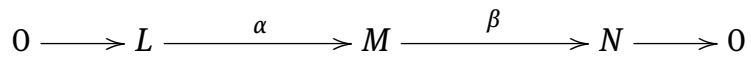


In this subsection we are going to verify that $\widetilde{\chi}(M) \geq \widetilde{\chi}(N)+\widetilde{\chi}(L)$. We proceed by steps:

Step 1. Let us verify that $\widetilde{\chi}(M) \geq \max \{\widetilde{\chi}(N), \widetilde{\chi}(L)\}$. In fact, any finitely generated submodule of $L$ is also a finitely generated submodule of $M$ so that, clearly, $\widetilde{\chi}(L) \leq \widetilde{\chi}(M)$. On the other hand, given a finitely generated submodule $\bar{F} \leq N$, there is a finitely generated submodule $F \leq M$ such that $\beta(F)=\bar{F}$ and, by the monotonicity of $\bar{\chi}$ under taking quotients, $\widetilde{\chi}(M) \geq \bar{\chi}(F) \geq \bar{\chi}(\bar{F})$. As this holds for any finitely generated $\bar{F} \leq N$, we conclude that $\widetilde{\chi}(M) \geq \widetilde{\chi}(N)$.

Step 2. Let us verify the statement in case $M \in \mathrm{fp}(R)$. Indeed, for any finitely generated $L^{\prime} \leq L$, consider the following short exact sequence: $0 \rightarrow L^{\prime} \rightarrow M \rightarrow M / L^{\prime} \rightarrow 0$. Then, $M$ and $M / L^{\prime}$ do belong to $\mathrm{fp}(R)$ and we can apply Lemma 1.1 (2) and the above Step 1 to get

$$
\widetilde{\chi}\left(L^{\prime}\right)=\widetilde{\chi}(M)-\widetilde{\chi}\left(M / L^{\prime}\right) \leq \widetilde{\chi}(M)-\widetilde{\chi}(N) .
$$

As this holds for any f.g. $L^{\prime} \leq L$, we conclude that $\widetilde{\chi}(L) \leq \widetilde{\chi}(M)-\widetilde{\chi}(N)$.

Step 3. Let us verify the statement in case $M \in \mathrm{fg}(R)$ (so that also $N \in \mathrm{fg}(R)$ ). Indeed, consider a surjection $M^{\prime} \rightarrow M$ with $M^{\prime} \in \mathrm{fp}(R)$ and complete to the following pullback diagram

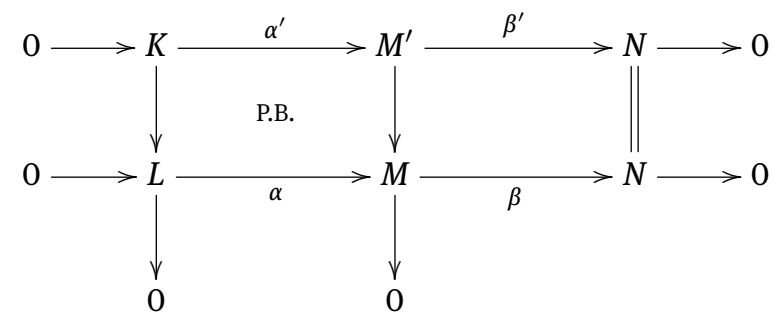

Then, $\widetilde{\chi}\left(M^{\prime}\right) \geq \widetilde{\chi}(N)+\widetilde{\chi}(K)$ by Step 2 , and $\widetilde{\chi}(K) \geq \widetilde{\chi}(L)$ by Step 1 . Thus, $\widetilde{\chi}\left(M^{\prime}\right) \geq \widetilde{\chi}(N)+\widetilde{\chi}(L)$. As this holds for any surjection $M^{\prime} \rightarrow M$ with $M^{\prime} \in \mathrm{fp}(R)$, we get $\widetilde{\chi}(M) \geq \widetilde{\chi}(N)+\widetilde{\chi}(L)$.

Step 4. We can now conclude easily as follows: let $L^{\prime} \leq L$ and $N^{\prime} \leq N$ be finitely generated submodules. Then there exists $M^{\prime} \leq M$ finitely generated such that $\beta\left(M^{\prime}\right)=N^{\prime}$ and $L^{\prime} \leq M^{\prime}$. Thus we have a short exact sequence $0 \rightarrow M^{\prime} \cap L \rightarrow M^{\prime} \rightarrow N^{\prime} \rightarrow 0$ with $M^{\prime} \in \operatorname{fg}(R)$ and Steps 1 and 3 tell us that

$$
\widetilde{\chi}(M) \geq \widetilde{\chi}\left(M^{\prime}\right) \geq \widetilde{\chi}\left(M^{\prime} \cap L\right)+\widetilde{\chi}\left(N^{\prime}\right) \geq \widetilde{\chi}\left(L^{\prime}\right)+\widetilde{\chi}\left(N^{\prime}\right) .
$$

As $L^{\prime}$ and $N^{\prime}$ were chosen arbitrarily, we get $\widetilde{\chi}(M) \geq \widetilde{\chi}(L)+\widetilde{\chi}(N)$.

\section{$1.4 \widetilde{\chi}$ is sub-additive}

Consider a short exact sequence in $R$-Mod as follows:

$$
0 \longrightarrow L \longrightarrow M \longrightarrow N \longrightarrow 0
$$

In this subsection we are going to verify that $\widetilde{\chi}(M) \leq \widetilde{\chi}(N)+\widetilde{\chi}(L)$. We proceed by steps:

Step 1. We start showing the statement in case $L$ and $N$ are finitely generated and the sequence splits (so $M \cong L \oplus N)$. Indeed, given $\varepsilon>0$, consider two surjections $L^{\prime} \rightarrow L$ and $N^{\prime} \rightarrow N$ with $L^{\prime}, N^{\prime} \in \operatorname{fp}(R)$, and such that $\widetilde{\chi}\left(L^{\prime}\right) \leq \widetilde{\chi}(L)+\varepsilon / 2$ and $\widetilde{\chi}\left(N^{\prime}\right) \leq \widetilde{\chi}(N)+\varepsilon / 2$. Then, there is a surjection $L^{\prime} \oplus N^{\prime} \rightarrow L \oplus N \cong M$ and $L^{\prime} \oplus N^{\prime} \in \mathrm{fp}(R)$, so that

$$
\widetilde{\chi}(M) \leq \widetilde{\chi}\left(L^{\prime} \oplus N^{\prime}\right)=\widetilde{\chi}\left(L^{\prime}\right)+\widetilde{\chi}\left(N^{\prime}\right) \leq \widetilde{\chi}(L)+\widetilde{\chi}(N)+\varepsilon
$$

where the equality is a consequence of (Mod.rk.2). We can conclude by the arbitrariness of $\varepsilon$. 
Step 2. Let us prove the statement in case $M \in \mathrm{fg}(R)$ and $N \in \mathrm{fp}(R)$. Indeed, consider a surjection $M^{\prime} \rightarrow M$ with $M^{\prime} \in \mathrm{fp}(R)$ and complete to the following pullback diagram:

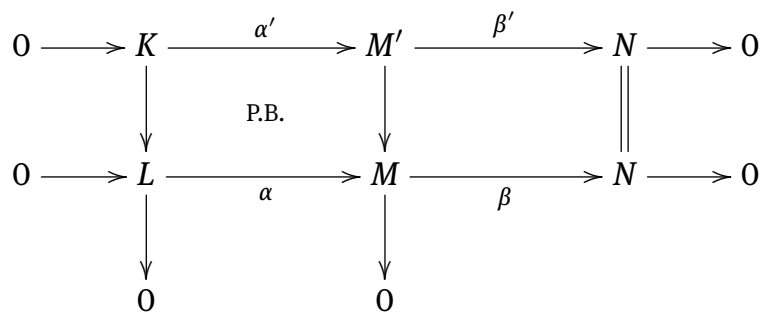

Now, by Step 1, $\widetilde{\chi}(L)+\widetilde{\chi}\left(M^{\prime}\right) \geq \widetilde{\chi}\left(L \oplus M^{\prime}\right)$, while, using the short exact sequence $0 \rightarrow K \rightarrow M^{\prime} \oplus L \rightarrow M \rightarrow 0$ and the super-additivity of $\widetilde{\chi}$, we get

$$
\widetilde{\chi}(L)+\widetilde{\chi}\left(M^{\prime}\right) \geq \widetilde{\chi}\left(L \oplus M^{\prime}\right) \geq \widetilde{\chi}(K)+\widetilde{\chi}(M) .
$$

Finally, notice that $\widetilde{\chi}\left(M^{\prime}\right)=\widetilde{\chi}(K)+\widetilde{\chi}(N)$ by super-additivity since $M^{\prime}, N \in \mathrm{fp}(R)$. Thus, $\widetilde{\chi}(L)+\widetilde{\chi}(K)+\widetilde{\chi}(N) \geq$ $\widetilde{\chi}(K)+\widetilde{\chi}(M)$, and so $\widetilde{\chi}(L)+\widetilde{\chi}(N) \geq \widetilde{\chi}(M)$.

Step 3. Let us prove the statement in case $N \in \mathrm{fp}(R)$. Indeed, given a finitely generated submodule $M^{\prime} \leq M$ there exists another finitely generated submodule $M^{\prime \prime} \leq M$ such that $M^{\prime} \leq M^{\prime \prime}$ and $\beta\left(M^{\prime \prime}\right)=N$. Then we can apply Step 2 to the short exact sequence $0 \rightarrow L \cap M^{\prime \prime} \rightarrow M^{\prime \prime} \rightarrow N \rightarrow 0$ to get (using also the already proved monotonicities of $\widetilde{\chi}$ )

$$
\widetilde{\chi}\left(M^{\prime}\right) \leq \widetilde{\chi}\left(M^{\prime \prime}\right) \leq \widetilde{\chi}\left(L \cap M^{\prime \prime}\right)+\widetilde{\chi}(N) \leq \widetilde{\chi}(L)+\widetilde{\chi}(N) .
$$

One now concludes by the arbitrariness of $M^{\prime}$.

Step 4. Let us prove the statement in case $M \in \mathrm{fg}(R)$ (so that also $N \in \mathrm{fg}(R)$ ). Given $\varepsilon>0$, take a surjection $N^{\prime} \rightarrow N$ with $N^{\prime} \in \operatorname{fp}(R)$ and such that $\widetilde{\chi}\left(N^{\prime}\right) \leq \widetilde{\chi}(N)+\varepsilon$, and consider the following pullback diagram:

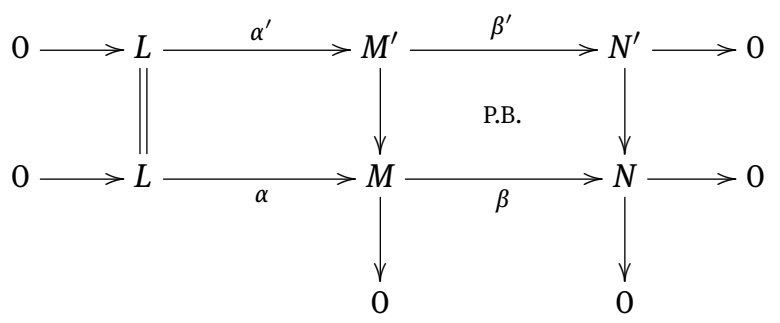

Then, by the usual monotonicities and Step $3, \widetilde{\chi}(M) \leq \widetilde{\chi}\left(M^{\prime}\right) \leq \widetilde{\chi}\left(N^{\prime}\right)+\widetilde{\chi}(L) \leq \widetilde{\chi}(N)+\widetilde{\chi}(L)+\varepsilon$. One concludes by the arbitrariness of $\varepsilon$.

Step 5. In the general case, consider a finitely generated submodule $M^{\prime} \leq M$ and notice that, by the already proved monotonicities and applying Step 4 to the short exact sequence $0 \rightarrow M^{\prime} \cap L \rightarrow M^{\prime} \rightarrow \beta\left(M^{\prime}\right) \rightarrow 0$, we get

$$
\widetilde{\chi}\left(M^{\prime}\right) \leq \widetilde{\chi}\left(\beta\left(M^{\prime}\right)\right)+\widetilde{\chi}\left(M^{\prime} \cap L\right) \leq \widetilde{\chi}(L)+\widetilde{\chi}(N) .
$$

One concludes by the arbitrariness of $M^{\prime}$.

\section{References}

[1] William Crawley-Boevey. Additive functions on locally finitely presented Grothendieck categories. Comm. Algebra, 22(5):1629-1639, 1994.

[2] William Crawley-Boevey. Locally finitely presented additive categories. Comm. Algebra, 22(5):1641-1674, 1994.

[3] Kenneth R Goodearl. Von Neumann regular rings, volume 4. Pitman London, 1979. 
[4] Andrei Jaikin-Zapirain. L2-betti numbers and their analogues in positive characteristic. Groups St Andrews 2017 in Birmingham, 455:346, 2019.

[5] D. G. Northcott and M. Reufel. A generalization of the concept of length. Quart. J. Math. Oxford Ser. (2), 16:297-321, 1965.

[6] Aidan Harry Schofield. Representations of rings over skew fields, volume 92. Cambridge University Press, 1985.

[7] Peter Vámos. Additive functions and duality over Noetherian rings. Quart. J. Math. Oxford Ser. (2), 19:43-55, 1968.

[8] Peter Vámos. Length Functions on Modules. PhD thesis, University of Sheffield, 1968. 